\title{
Some properties of the fractional circle Zernike polynomials*
}

\author{
M.M. Rodrigues ${ }^{\ddagger} \quad$ N. Vieira ${ }^{\ddagger}$ \\ † School of Technology and Management, \\ Polytechnic Institute of Leiria \\ P-2411-901, Leiria, Portugal. \\ ¥ CIDMA - Center for Research and Development in Mathematics and Applications \\ Department of Mathematics, University of Aveiro \\ Campus Universitário de Santiago, 3810-193 Aveiro, Portugal. \\ E-mails: mrodrigues@ua.pt,nloureirovieira@gmail.com
}

\begin{abstract}
In this paper, we present a fractional extension of the classical circle Zernike polynomials defined via $\mathrm{g}$-Jacobi functions. Some properties of this new class of functions are studied, such as recurrence relations for consecutive and distant neighborhoods, and differential relations. A graphic representation for the proposed fractional circle Zernike polynomials will be presented in the final section of the paper.

Keywords: Zernike polynomials; Fractional calculus, g-Jacobi polynomials; Special functions; Recurrence relations; Rodrigues and operational definition of circle polynomials.
\end{abstract}

MSC 2010: 26A33; 78M99; 33C47.

\section{Introduction}

The cornea is the major refracting component of the human eye, contributing approximately two thirds of the eye optical power. There are many types of mathematical models for corneal topography. The most common and simple models are based on conic sections [5], or on generalized conic functions [11]. Currently, the Zernike polynomials are the standard functions for the description of the wave front aberrations of the human eye and have been also used in the modeling of cornea surfaces [4].

The aim of this paper is to introduce the so-called fractional circle Zernike polynomials and study some of their properties. The outline of the paper reads as follows: In Section 2, we recall some basic notions about circle Zernike polynomials, fractional calculus and g-Jacobi functions. Then, we introduce the fractional circle Zernike polynomials and the explicit formulas for these new polynomials will be present, as well as the study of the existence of a continuous orthogonality relation will be done. Moreover, we present some recurrence relations for consecutive and distant neighborhoods, and we study the differential properties of these fractional polynomials. In the last section, we present a graphical representation for some fractional circle Zernike polynomials.

${ }^{*}$ The final version is published in Integral Methods in Science and Engineering-Vol.1, Eds: C. Constanda, P.D. Lamberti, P. Musolino and M.D. Riva, Birkäuser, Basel, (2017), 265-276. It as available via the website https://doi.org/10.1007/978-3-31959384-5_24 


\section{Preliminaries}

We start recalling some basic facts about circle Zernike polynomials which are studied in several references, see for instance [3]. The odd and even Zernike polynomials are given by (see [8])

$$
\begin{aligned}
Z_{n}^{-m}(\rho, \theta) & ={ }^{o} U_{n}^{m}(\rho, \theta)=R_{n}^{m}(\rho) \sin (m \theta), \\
Z_{n}^{m}(\rho, \theta) & ={ }^{e} U_{n}^{m}(\rho, \theta)=R_{n}^{m}(\rho) \cos (m \theta),
\end{aligned}
$$

where $\theta$ is the azimuthal angle with $0 \leq \theta \leq 2 \pi, \rho$ is the radial distance with $0 \leq \rho \leq 1$, and the radial function $R_{n}^{m}(\rho)$ is defined for $n, m \in \mathbb{N}_{0}$, with $m \leq n$ by

$$
R_{n}^{m}(\rho)= \begin{cases}\sum_{k=0}^{\frac{n-m}{2}} \frac{(-1)^{k}(n-k) !}{k !\left(\frac{n+m}{2}-k\right) !\left(\frac{n-m}{2}-k\right) !} \rho^{n-2 k}, & \text { for } n-m \text { even } \\ 0, & \text { for } n-m \text { odd }\end{cases}
$$

When $n-m$ is even, the radial functions $R_{n}^{m}(\rho)$ can be written in terms of the Jacobi polynomial $P_{n}^{(\alpha, \beta)}(x)$ as

$$
R_{n}^{m}(\rho)=(-1)^{\frac{n-m}{2}} \rho^{m} P_{\frac{n-m}{2}}^{(m, 0)}\left(1-2 \rho^{2}\right)
$$

Now we recall the definitions of Riemann-Liouville fractional integral and fractional derivative in the sense of Riemann-Liouville (see $[7,10])$.

Definition 2.1 For $t>0$

$$
J^{\nu} f(t):=\frac{1}{\Gamma(\nu)} \int_{0}^{t}(t-\tau)^{\nu-1} f(\tau) d \tau
$$

is called the Riemann-Liouville fractional integral of the function $f(t)$ of order $\nu \in \mathbb{C}$ with $\operatorname{Re}(\nu)>0$.

Definition 2.2 If $t>0$ and $m \in \mathbb{N}_{0}$ such that $m-1 \leq \mu<m$, then the Riemann-Liouville fractional derivative of $f(t)$ of order $\mu$ is defined as

$$
D^{\mu} f(t)=D^{m}\left[J^{m-\mu} f(t)\right]
$$

(if it exists) where $m-\mu>0$.

Now, we pass to the definition and basic properties of the g-Jacobi functions introduced and studied in [6]. These functions will play a key role in the definition of the fractional Zernike polynomials, and it correspond to a generalization for the fractional case of the classical Jacobi polynomials.

Definition 2.3 We define the (generalized or) g-Jacobi functions by the formula

$$
P_{\nu}^{(\alpha, \beta)}(t)=(-2)^{-\nu} \Gamma(\nu+1)^{-1}(1-t)^{-\alpha}(1+t)^{-\beta} D^{\nu}\left[(1-t)^{\nu+\alpha}(1+t)^{\nu+\beta}\right]
$$

where $\nu \in \mathbb{R}^{+}, \alpha>-1, \beta>-1$ and $D^{\nu}$ is the Riemann-Liouville fractional differential operator (1).

In the following results we recall some properties of the g-Jacobi functions (see [6]), which are analogous to the corresponding properties of the classical Jacobi polynomials. In the first two results we give the corresponding explicit formulas for these functions.

Theorem 2.4 (Explicit formula) For the g-Jacobi functions holds the explicit formula

$$
P_{\nu}^{(\alpha, \beta)}(t)=2^{-\nu} \sum_{k=0}^{\infty}\left(\begin{array}{c}
\nu+\alpha \\
\nu-k
\end{array}\right)\left(\begin{array}{c}
\nu+\beta \\
k
\end{array}\right)(t-1)^{k}(t+1)^{\nu-k}
$$

where

$$
\left(\begin{array}{c}
\alpha \\
\beta
\end{array}\right)=\frac{\Gamma(1+\alpha)}{\Gamma(1+\beta) \Gamma(1+\alpha-\beta)}
$$

is the binomial coefficient with real arguments. 
Theorem 2.5 (Explicit formula) The g-Jacobian functions can be represented as

$$
\begin{aligned}
P_{\nu}^{(\alpha, \beta)}(t) & =\left(\begin{array}{c}
\nu+\alpha \\
\nu
\end{array}\right){ }_{2} F_{1}\left(-\nu, \nu+\alpha+\beta+1 ; \alpha+1 ; \frac{1-t}{2}\right) \\
& =\frac{1}{\Gamma(1+\nu)} \sum_{k=0}^{\infty}\left(\begin{array}{c}
\nu \\
k
\end{array}\right) \frac{\Gamma(1+\nu+\alpha+\beta+k) \Gamma(1+\alpha+\nu)}{\Gamma(1+\nu+\alpha+\beta) \Gamma(1+\alpha+k)}\left(\frac{t-1}{2}\right)^{k},
\end{aligned}
$$

where ${ }_{2} F_{1}(a, b ; c ; t)$ is the Gauss hypergeometric function.

In the following results we will present the most basic properties of the g-Jacobi functions.

Theorem 2.6 (Differential equation) The g-Jacobi functions satisfy the linear homogeneous differential equation of second order

$$
(1-t)^{2} y^{\prime \prime}+[\beta-\alpha-(\alpha+\beta+2) t] y^{\prime}+\nu(\nu+\alpha+\beta+1) y=0,
$$

or

$$
D_{t}\left\{(1-t)^{\alpha+1}(1+t)^{\beta+1} y^{\prime}\right\}+\nu(\nu+\alpha+\beta+1)(1-t)^{\alpha}(1+t)^{\beta} y=0 .
$$

Theorem 2.7 The g-Jacobi functions satisfy the following properties (with $n \in \mathbb{N}$ ):

1. $\lim _{\nu \rightarrow n} P_{\nu}^{(\alpha, \beta)}(t)=P_{n}^{(\alpha, \beta)}(t) ;$

2. $P_{\nu}^{(\alpha, \beta)}(-t)=(-1)^{\nu} P_{\nu}^{(\alpha, \beta)}(t)$;

3. $P_{\nu}^{(\alpha, \beta)}(1)=\left(\begin{array}{c}\nu+\alpha \\ \nu\end{array}\right)$;

4. $P_{\nu}^{(\alpha, \beta)}(-1)=\left(\begin{array}{c}\nu+\beta \\ \nu\end{array}\right)$;

5. $D_{t} P_{\nu}^{(\alpha, \beta)}(t)=\frac{1}{2}(\nu+\alpha+\beta+1) P_{\nu-1}^{(\alpha+1, \beta+1)}(t)$.

\section{Fractional circle Zernike polynomials}

The aim of this section is to introduce the so-called fractional circle Zernike polynomials and study some of their main properties. Taking into account the definition of g-Jacobi functions presented previously and the ideas presented in [3], we introduce the definition for the fractional circle Zernike polynomials.

Definition 3.1 We define the fractional circle Zernike polynomials by

$$
\begin{gathered}
Z_{\nu}^{-m}(\rho, \theta)=R_{\nu}^{m}(\rho) \sin (m \theta), \quad \text { when }[\nu-m] \text { is even } \\
Z_{\nu}^{m}(\rho, \theta)=R_{\nu}^{m}(\rho) \cos (m \theta), \quad \text { when }[\nu-m] \text { is odd }
\end{gathered}
$$

where $m \in \mathbb{N}_{0}, \nu \in \mathbb{R}_{+}, \nu>m, 0 \leq \rho \leq 1$ is the radial distance, $0 \leq \theta \leq 2 \pi$ is the azimuthal angle, and $[\beta]$ represents the integer part of $\beta$. The fractional radial function $R_{\nu}^{m}(\rho)$ will be defined as

$$
R_{\nu}^{m}(\rho)=(-1)^{\left[\frac{\nu-m}{2}\right]} \rho^{m} P_{\frac{\nu-m}{2}}^{(m, 0)}\left(1-2 \rho^{2}\right)
$$

where $P_{\nu}^{(\alpha, \beta)}(x)$ are the $g$-Jacobi functions defined in (1).

Taking into account the properties of the g-Jacobi functions introduced in [6], we derive the following results. 
Theorem 3.2 (Explicit formula) The fractional radial function $R_{\nu}^{m}(\rho)$ allows the following explicit formulas

$$
\begin{aligned}
R_{\nu}^{m}(\rho) & =\sum_{k=0}^{\infty}\left(\begin{array}{c}
\frac{\nu+m}{2} \\
\frac{\nu-m}{2}+k
\end{array}\right)\left(\begin{array}{c}
\frac{\nu-m}{2} \\
k
\end{array}\right)(-1)^{\left[\frac{v-m}{2}\right]+k} 2^{\frac{\nu-m}{2}} \rho^{2 k+m}\left(1-\rho^{2}\right)^{\frac{\nu-m}{2}-k} \\
& =\frac{1}{\Gamma\left(1+\frac{\nu-m}{2}\right)} \sum_{k=0}^{\infty}\left(\begin{array}{c}
\frac{\nu-m}{2} \\
k
\end{array}\right) \frac{\Gamma\left(1+k+\frac{\nu+m}{2}\right)}{(m+k) !}(-1)^{\left[\frac{\nu-m}{2}\right]+k} \rho^{m+2 k},
\end{aligned}
$$

where

$$
\left(\begin{array}{c}
\alpha \\
\beta
\end{array}\right)=\frac{\Gamma(1+\alpha)}{\Gamma(1+\beta) \Gamma(1+\alpha-\beta)} .
$$

Proof: The result is obtained by straightforward calculations based on the substitution of (4) into the explicit formulas (2), (3), respectively.

Now, we present some recurrence relations for $R_{\nu}^{m}(\rho)$. We will split our relations in two results. The first one refers to the recurrence relations for the consecutive neighborhoods, and the second for distant neighborhoods.

Theorem 3.3 The radial function $R_{\nu}^{m}(\rho)$ verifies the following recurrence relations for consecutive neighborhoods:

$$
\begin{aligned}
R_{\nu}^{m}(\rho) & =\frac{-2 \nu \rho}{\nu-m} R_{\nu-1}^{m-1}(\rho)+R_{\nu-2}^{m}(\rho) \\
R_{\nu}^{m}(\rho) & =\frac{2(\nu+2)}{\nu-m+2} \rho R_{\nu-1}^{m-1}(\rho)+\frac{\nu+m+2}{\nu-m+2} R_{\nu+2}^{m}(\rho) ; \\
R_{\nu}^{m}(\rho) & =-\frac{\nu-m+2}{\nu+m+2} R_{\nu+2}^{m}(\rho)-\frac{2(\nu+2)}{\nu+m+2} \rho R_{\nu-1}^{m-1}(\rho) ; \\
R_{\nu}^{m}(\rho) & =\frac{2(\nu+3)\left[m^{2}+\left(1-2 \rho^{2}\right)(\nu+2)(\nu+4)\right]}{(\nu+m+2)(\nu-m+2)(\nu+4)} R_{\nu+2}^{m}(\rho)-\frac{(\nu-m)(\nu+m+4)(\nu+2)}{(\nu+m+2)(\nu-m+2)(\nu+4)} R_{\nu+4}^{m}(\rho) \\
R_{\nu}^{m}(\rho) & =\frac{2(\nu-1)\left[m^{2}+\nu\left(1-2 \rho^{2}\right)(\nu-2)\right]}{\left(\nu^{2}-m^{2}\right)(\nu-2)} R_{\nu-2}^{m}(\rho)-\frac{\nu(\nu+m-2)(\nu-m-1)}{\left(\nu^{2}-m^{2}\right)(\nu-2)} R_{\nu-4}^{m}(\rho) ; \\
R_{\nu}^{m}(\rho) & =\frac{2(1+\rho)-m(2+\rho)-\rho \nu(1+2 \rho)}{\rho(\nu+m)} R_{\nu-1}^{m-1}(\rho) ; \\
\rho R_{\nu}^{m}(\rho) & =\frac{2 m\left(1+\rho^{2}\right)-2\left(m-2 \rho^{2}-\nu-1\right)}{\nu+m-2} R_{\nu+1}^{m+1}(\rho)-\frac{\rho(\nu+m+4)}{\nu+m+2} R_{\nu+2}^{m+2}(\rho)
\end{aligned}
$$

Proof: The proof of the recurrence relations takes into account the recurrence relations presented in [1]Chapter 22 and [9]-Appendix II.11 for the classical Jacobi polynomials, the definition of g-Jacobi functions presented in the Preliminaries, and relation (4). Here we only present the proof for the first relation since the others are similar. From relation (22.7.18) in [1] we have

$$
\nu P_{\frac{\nu-m}{2}}^{(m-1,0)}\left(1-2 \rho^{2}\right)=\frac{\nu+m}{2} P_{\frac{\nu-m}{2}}^{(m, 0)}\left(1-2 \rho^{2}\right)-\frac{\nu-m}{2} P_{\frac{\nu-m}{2}-1}^{(m, 0)}\left(1-2 \rho^{2}\right)
$$

for $m \in \mathbb{N}_{0}, \nu \in \mathbb{R}_{+}$with $\nu>m$, and $0 \leq \rho \leq 1$. On the other hand, from relation (4) we get

$$
P_{\frac{v-m}{2}}^{(m, 0)}\left(1-2 \rho^{2}\right)=(-1)^{\left[\frac{v-m}{2}\right]} \rho^{-m} R_{\nu}^{m}(\rho) .
$$

Combining (5) and (6) we obtain

$$
\frac{\nu-m}{2} R_{\nu}^{m}(\rho)=-\nu \rho R_{\nu-1}^{m-1}(\rho)+\frac{\nu-m}{2} R_{\nu-2}^{m}(\rho),
$$

which is equivalent to the first relation.

Theorem 3.4 For $N \in \mathbb{N}$, the radial function $R_{\nu}^{m}(\rho)$ verifies the following recurrence relations for distant neighborhoods: 
1. Forward case

$$
R_{\nu}^{m}(\rho)=C_{N} R_{\nu+2 N}^{m}(\rho)-\frac{(2 N+\nu-m+2)(2 N+\nu+m+2)(2 N+\nu)}{(N+\nu+m)(2 N+\nu-m)(2 N+\nu+2)} C_{N-1} R_{\nu+2 N+2}^{m}(\rho) ;
$$

where $C_{N}$ and $C_{N-1}$ depend on the parameters $\nu, m$ and $\rho$, and are such that

$$
\begin{aligned}
C_{0}= & 1 ; \\
C_{1}= & \frac{2(\nu+3)\left[m^{2}+\left(1-2 \rho^{2}\right)(\nu+2)(\nu+4)\right]}{(\nu+m+2)(\nu-m+2)(\nu+4)} ; \\
C_{N}= & \frac{2(2 N+\nu+1)\left[m^{2}+(2 N+\nu+2)\left(1-2 \rho^{2}\right)(2 N+\nu)\right]}{(2 N+\nu+m)(2 N+\nu-m)(2 N+\nu+2)} C_{N-1} \\
& -\frac{(2 N+\nu-m)(2 N+\nu+m)(2 N+\nu-2)}{(2 N+\nu+m-2)(2 N+\nu-m-2)(2 N+\nu)} C_{N-2} ;
\end{aligned}
$$

2. Backward case

$$
R_{\nu}^{m}(\rho)=D_{N} R_{\nu-2 N}^{m}(\rho)+\frac{(\nu+m-2 N)(\nu-m-2 N)(\nu+2-2 N)}{(2 N-\nu+m-2)(\nu+m+2-2 N)(\nu-2 n)} D_{N-1} R_{\nu-2 N-2}^{m}(\rho)
$$

where $D_{N}$ and $D_{N-1}$ depend on the parameters $\nu, m$ and $\rho$, and are such that

$$
\begin{aligned}
D_{0}= & 1 ; \\
D_{1}= & \frac{2(\nu-1)\left[m^{2}+\nu\left(1-2 \rho^{2}\right)(\nu-2)\right]}{\left(\nu^{2}-m^{2}\right)(\nu-2)} ; \\
D_{N}= & \frac{2(\nu+1-2 N)\left[m^{2}+(\nu-2 N)\left(1-2 \rho^{2}\right)(\nu+2-2 N)\right]}{(\nu-m+2-2 N)(\nu+m+2-2 N)(\nu-2 N)} D_{N-1} \\
& +\frac{(\nu+m+2-2 N)(\nu-m+2-2 N)(\nu+4-2 N)}{(2 N-\nu+m-4)(\nu+m+4-2 N)(\nu+2-2 N)} D_{N-2 .} .
\end{aligned}
$$

The proof will be omitted since it follows the same ideas used in the proof of the previous theorem. Finally, we introduce some differential properties of the fractional circle Zernike polynomials $Z_{\nu}^{ \pm m}(\rho, \theta)$.

Theorem 3.5 The radial function $R_{\nu}^{m}(\rho)$ verifies the following partial differential relation

$$
\rho D_{\rho} R_{\nu}^{m}(\rho)=\left[m-(\nu-m)\left(m-\nu\left(1-2 \rho^{2}\right)\right)\right] R_{\nu}^{m}(\rho)-\frac{2(\nu+m)}{\nu} R_{\nu-2}^{m}(\rho)
$$

Proof: From relation (22.8.1) in [1] we have

$$
2 \nu \rho^{2} D_{\rho} P_{\frac{\nu-m}{2}}^{(m, 0)}\left(1-2 \rho^{2}\right)=\frac{\nu-m}{2}\left[m-\nu\left(1-2 \rho^{2}\right)\right] P_{\frac{\nu-m}{2}}^{(m, 0)}\left(1-2 \rho^{2}\right)+\frac{\nu^{2}-m^{2}}{2} P_{\frac{\nu-m}{2}-1}^{(m, 0)}\left(1-2 \rho^{2}\right) .
$$

On the other hand, from (4) we have

$$
D_{\rho} P_{\frac{\nu-m}{2}}^{(m, 0)}\left(1-2 \rho^{2}\right)=\frac{(-1)^{\left[\frac{\nu-m}{2}\right]-1}}{4}\left(-m \rho^{-m-2} R_{\nu}^{m}\left(1-2 \rho^{2}\right)+\rho^{-m-1} D_{\rho} R_{\nu}^{m}\left(1-2 \rho^{2}\right)\right) .
$$

Combining (4), (7) and (8), and after straightforward calculations we obtain our result.

From the previous results follows immediately the next corollary.

Corollary 3.6 The fractional circle Zernike polynomials $Z_{\nu}^{ \pm m}(\rho, \theta)$ verify the following partial differential relation

$$
\rho D_{\rho} Z_{\nu}^{ \pm m}(\rho, \theta)=\left[m-(\nu-m)\left(m-\nu\left(1-2 \rho^{2}\right)\right)\right] Z_{\nu}^{ \pm m}(\rho, \theta)-\frac{2(\nu+m)}{\nu} Z_{\nu}^{ \pm m}(\rho, \theta) .
$$

Theorem 3.7 The fractional radial function $R_{\nu}^{m}(\rho)$ satisfies the following partial differential equation of second order

$$
\frac{\rho^{5}\left(1-\rho^{2}\right)}{4} D_{\rho}^{2} R_{\nu}^{m}(\rho)-\frac{\rho^{3}\left(3 \rho^{2}-1\right)}{4} D_{\rho} R_{\nu}^{m}(\rho)+\frac{\rho^{2}\left(\rho^{2} \nu(\nu+2)-m^{2}\right)}{4} R_{\nu}^{m}(\rho)=0
$$


Proof: Taking into account [6], the g-Jacobi function $P_{\frac{\nu-m}{2}}^{(m, 0)}(x)$ satisfies the following differential equation

$$
\left(1-x^{2}\right) D_{x}^{2} P_{\frac{\nu-m}{2}}^{(m, 0)}(x)-\left[m+(m+2)\left(1-2 \rho^{2}\right)\right] D_{x} P_{\frac{\nu-m}{2}}^{(m, 0)}(x)+\frac{\nu-m}{2}\left(\frac{\nu+m}{2}+1\right) P_{\frac{\nu-m}{2}}^{(m, 0)}(x)=0 .
$$

On the other hand, from relation (4) we obtain the following equalities

$$
\begin{aligned}
P_{\frac{\nu-m}{2}}^{(m, 0)}\left(1-2 \rho^{2}\right)= & (-1)^{\left[\frac{\nu-m}{2}\right]} \rho^{-m} R_{\nu}^{m}(\rho) ; \\
D_{\rho} P_{\frac{\nu-m}{2}}^{(m, 0)}\left(1-2 \rho^{2}\right)= & \frac{(-1)^{\left[\frac{\nu-m}{2}\right]}}{4}\left[-m \rho^{-m-2} R_{\nu}^{m}(\rho)+\rho^{-m-1} D_{\rho} R_{\nu}^{m}(\rho)\right] ; \\
D_{\rho}^{2} P_{\frac{\nu-m}{2}}^{(m, 0)}\left(1-2 \rho^{2}\right)= & \frac{(-1)^{\left[\frac{\nu-m}{2}\right]}}{16}\left[m(m+2) \rho^{-m-4} R_{\nu}^{m}(\rho)\right. \\
& \left.-(2 m+1) \rho^{-m-3} D_{\rho} R_{\nu}^{m}(\rho)+\rho^{-m-1} D_{\rho}^{2} R_{\nu}^{m}(\rho)\right] .
\end{aligned}
$$

Substituting (10), (11) and (12) into (9) and after straightforward calculations, we get

$$
0=\frac{\rho^{5}\left(1-\rho^{2}\right)}{4} D_{\rho}^{2} R_{\nu}^{m}(\rho)-\frac{\rho^{3}\left(3 \rho^{2}-1\right)}{4} D_{\rho} R_{\nu}^{m}(\rho)+\frac{\rho^{2}\left(\rho^{2} \nu(\nu+2)-m^{2}\right)}{4} R_{\nu}^{m}(\rho) .
$$

From the previous theorem follows immediately the following corollary.

Corollary 3.8 The fractional circle Zernike polynomials $Z_{\nu}^{ \pm m}(\rho, \theta)$ satisfy the following partial differential equation of second order

$$
\frac{\rho^{5}\left(1-\rho^{2}\right)}{4} D_{\rho}^{2} Z_{\nu}^{ \pm m}(\rho, \theta)-\frac{\rho^{3}\left(3 \rho^{2}-1\right)}{4} D_{\rho} Z_{\nu}^{ \pm m}(\rho, \theta)+\frac{\rho^{2}\left(\rho^{2} \nu(\nu+2)-m^{2}\right)}{4} Z_{\nu}^{ \pm m}(\rho, \theta)=0 .
$$

\section{Graphical representation of fractional circle Zernike polynomials}

The aim of this section is to present some graphical representation for the proposed fractional circle Zernike polynomials. In the literature (see [2]) we can find the well known correspondences between combinations of integer values of $\nu$ and $\mathrm{m}$ and some cornea aberrations:

\begin{tabular}{||c|c|c||}
\hline \hline Radial Degree $(\nu)$ & Azimuthal Degree $(m)$ & Cornea Aberration \\
\hline \hline 0 & 0 & Piston \\
\hline 1 & \pm 1 & Tip \\
\hline 2 & 0 & Defocus \\
\hline 2 & \pm 2 & Astigmatism \\
\hline 3 & \pm 1 & Coma \\
\hline 3 & \pm 3 & Trefoil \\
\hline 4 & 0 & Spherical Aberration \\
\hline \hline
\end{tabular}

In the following three groups of figures, we present a graphical representation of fractional circle Zernike polynomials for the cases of Defocus $(m=0)$, Astigmatism $(m=2)$ and Trefoil $(m=3)$, for different values of $\nu$ including the integer case. 

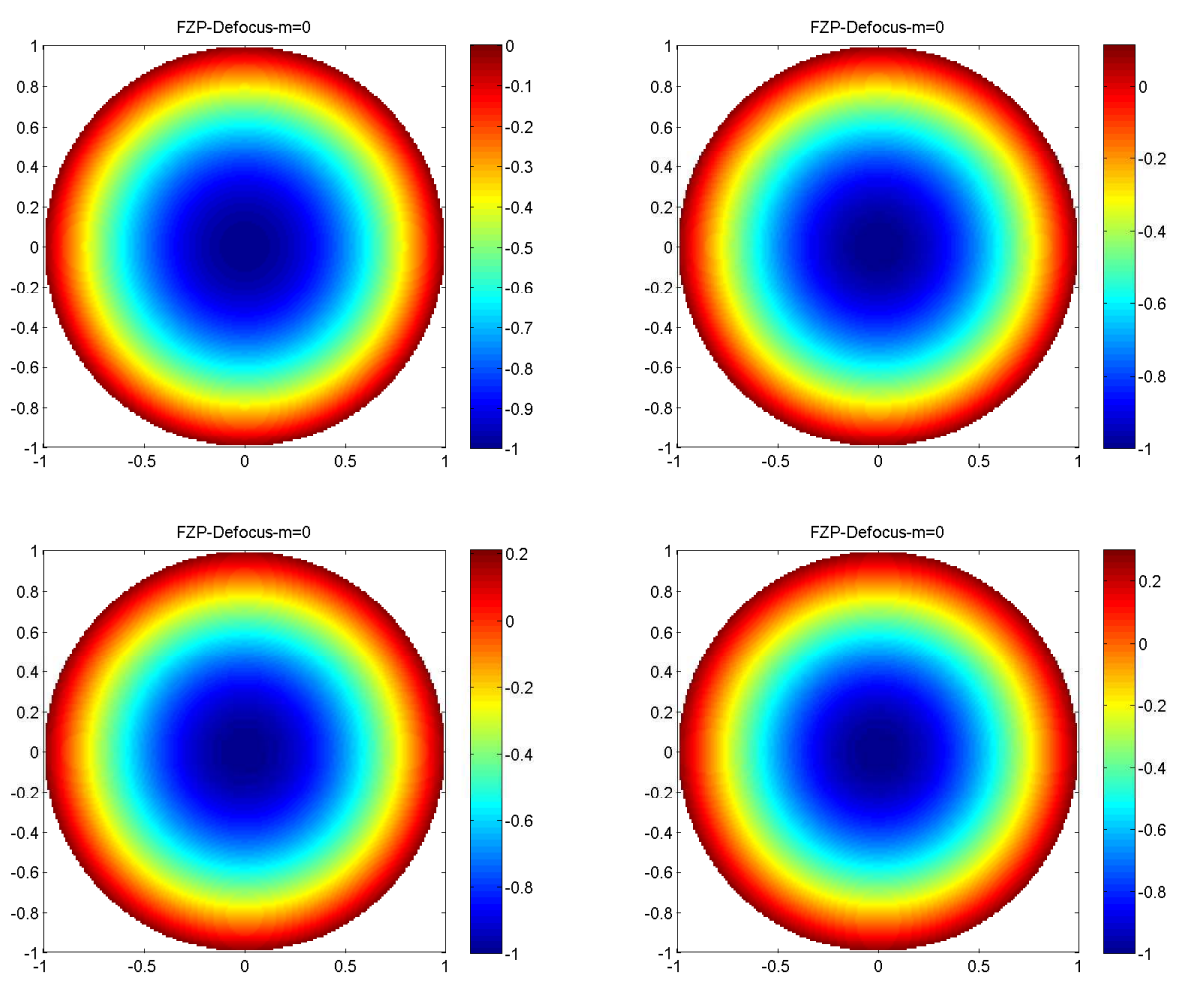

Figure 1: Fractional circle Zernike polynomials for Defocus considering $\nu=2.0$-plot $(1,1) ; \nu=2.33$-plot $(1,2)$; $\nu=2.66-\operatorname{plot}(2,1) ; \nu=2.99-\operatorname{plot}(2,2)$.
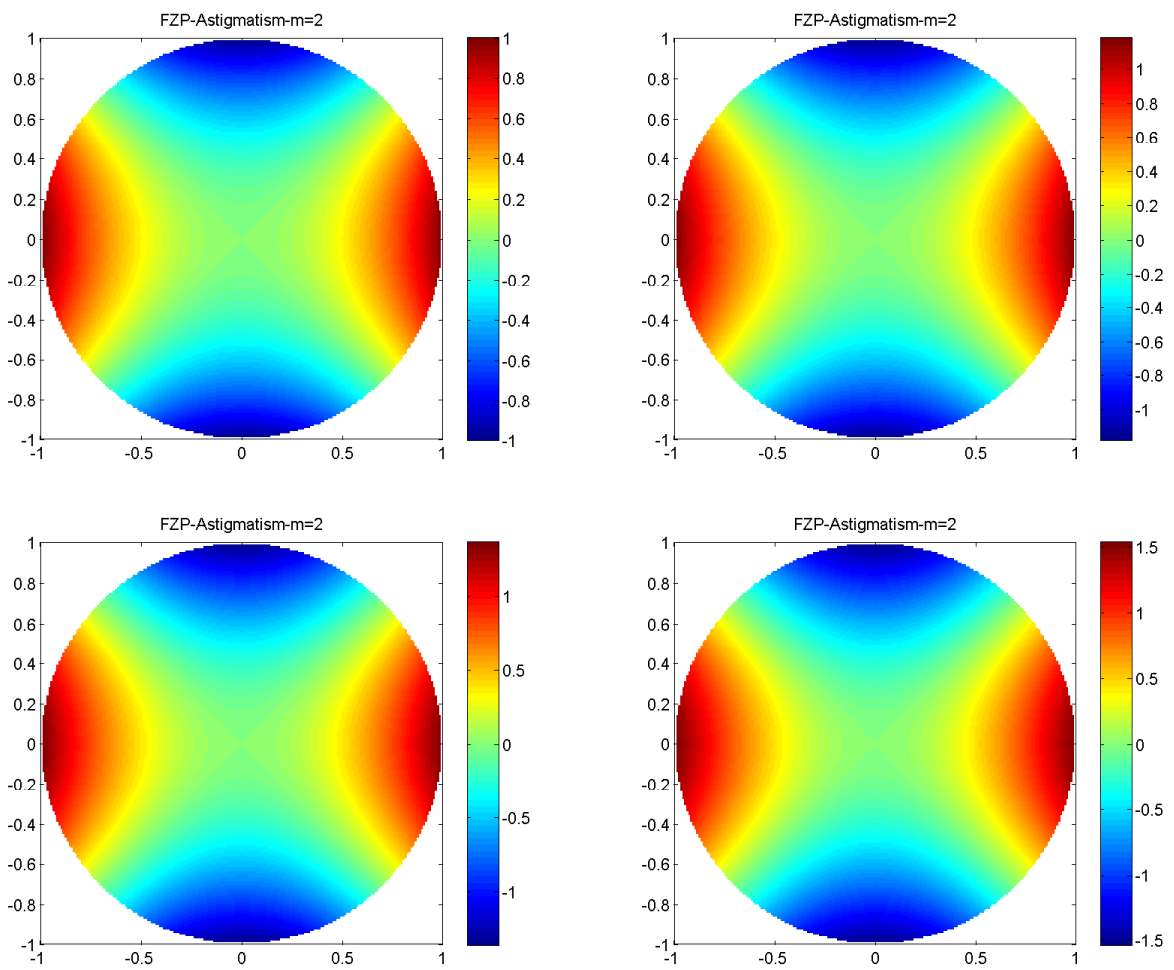

Figure 2: Fractional circle Zernike polynomials for Astigmatism considering $\nu=2.0-\operatorname{plot}(1,1) ; \nu=2.33$ $\operatorname{plot}(1,2) ; \nu=2.66-\operatorname{plot}(2,1) ; \nu=2.99-\operatorname{plot}(2,2)$. 

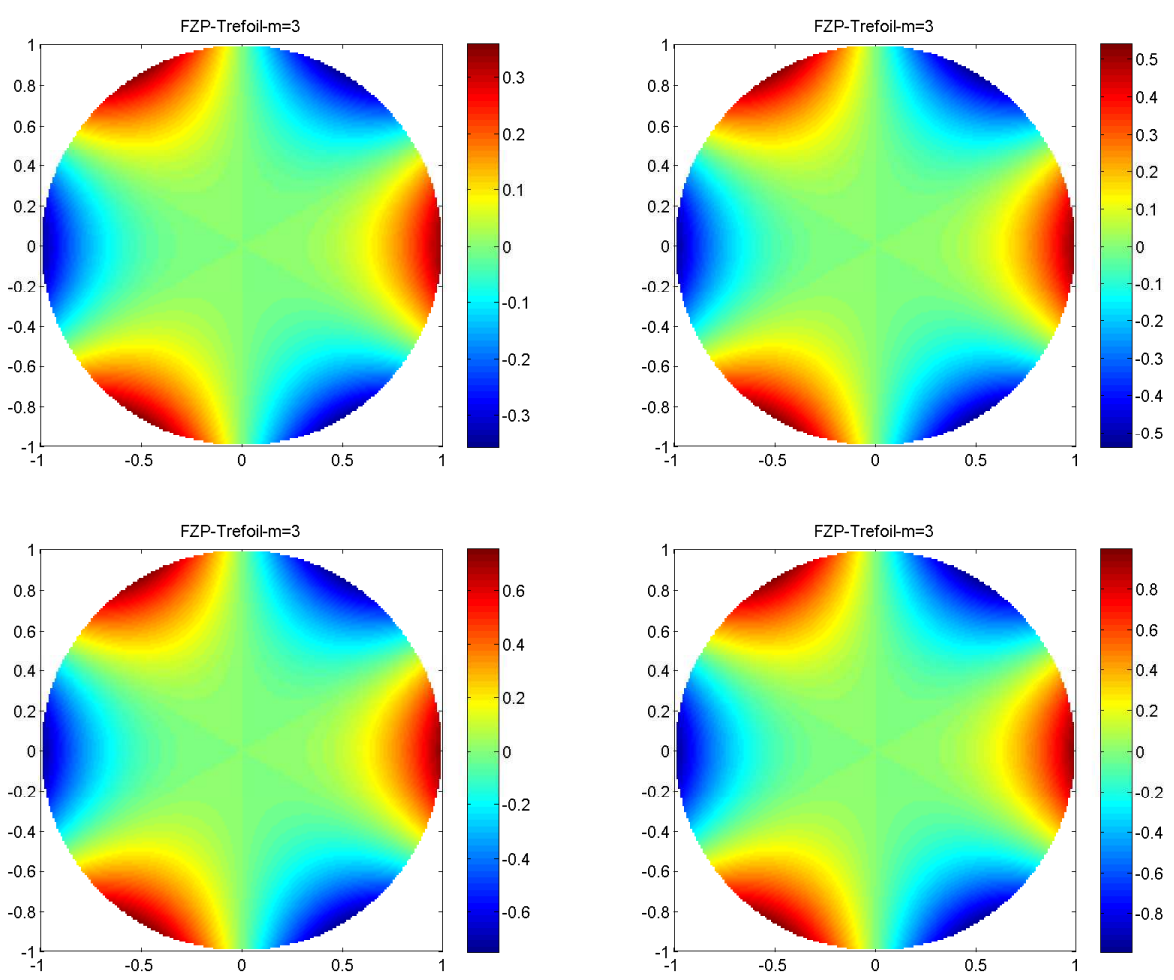

Figure 3: Fractional circle Zernike polynomials for Trefoil considering $\nu=3.0-\operatorname{plot}(1,1) ; \nu=3.33$-plot $(1,2)$; $\nu=3.66-\operatorname{plot}(2,1) ; \nu=3.99-\operatorname{plot}(2,2)$. 
From the three stated cases, we verify that the cornea aberration becomes more serious for values of $\nu$ far from the corresponding integer value already studied in the bibliography. This fact is supported by the increasing of the maximum value of the figure's scale. The authors believe that, this fact should have a medical interpretation and it could help in the study of each cornea aberration. Moreover, the analysis of the presented figures allows us to induce that this new class of fractional circle polynomials allow the possibility to establish a mathematically graduation (and therefore a exact medical graduation) for each cornea aberration. This fact will give the possibility of the development of new cornea models and accuracy of some medical surgical procedures and techniques used in the constructions of lens. We also remark that similar graphics can be obtained for the other cornea aberrations and for another fractional circle Zernike polynomials with $m \in \mathbb{N}_{0}$ and $\nu \in \mathbb{R}_{+}$.

Acknowledgement: The authors were supported by Portuguese funds through the CIDMA - Center for Research and Development in Mathematics and Applications, and the Portuguese Foundation for Science and Technology ("FCT-Fundaçâo para a Ciência e a Tecnologia"), within project UID/MAT/ 0416/2013. N. Vieira is Auxiliar Researcher, under the FCT Researcher Program 2014 (Ref: IF/00271/2014).

\section{References}

[1] M. Abramowitz and I.A. Stegun, Handbook of Mathematical Functions, U.S. G.P.O., Washington DC, 1972.

[2] M. Born and E. Wolf, The Diffraction Theory of Aberrations, In: Principles of Optics: Electromagnetic Theory of Propagation, Interference, and Diffraction of Light, 6th ed, Pergamon Press, New York, (1989), 459-490.

[3] M. Born and E. Wolf, Principles of Optics, Pergamon Press, London-New York-Paris-Los Angeles, 1959.

[4] D.R. Iskander, M.R. Morelande, M.J. Collins and B. Davis, Modeling corneal surfaces with radial polynomials, IEEE Trans. Biomed. Eng., 49-No.4, (2002), 320-328.

[5] H. Kasprzak and D.R. Iskander, Approximating ocular surfaces by generalized conic curves, Ophthal. Physiol. Opt., 26-No.6, (2006), 602-609.

[6] S.P. Mirevski, L. Boyadijiev and R. Scherer, On the Riemann-Liouville fractional calculus, g-Jabobi functions and F-Gauss functions, Appl. Math. Comput., 187-No.1, (2007), 315-325.

[7] I. Podlubny, Fractional Differential Equations. An introduction to fractional derivatives, fractional differential equations, to methods of their solution and some of their applications, Mathematics in Science and Engineering 198, Academic Press, San Diego, 1999.

[8] A. Prata and W.V.T. Rusch, Algorithm for computation of Zernike polynomials expansion coefficients, Appl. Opt., 28, (1989), 749-754.

[9] A.P. Prudnikov, Yu.A. Brychkov and O.I. Marichev, Integrals and Series, Volume 2: Special functions, Gordon and Breach Publisher, New York etc, 1988.

[10] S.G. Samko, A.A. Kilbas and O.I. Marichev, Fractional integrals and derivatives: theory and applications, Gordon and Breach, New York, 1993.

[11] J. Schwiegerling, J.E. Greivenkamp and J.M. Miller, Representation of videokeratoscopic height data with Zernike polynomials, J. Opt. Soc. Amer. A, 12-No.10, (1995), 2105-2113. 